\title{
FEEDBACK FROM NATURALISTIC DRIVING IMPROVES TREATMENT COMPLIANCE IN DRIVERS WITH OBSTRUCTIVE SLEEP APNEA
}

\author{
Jeffrey D. Dawson, ${ }^{1}$ Lixi Yu ${ }^{1}$, Nazan S. Aksan, ${ }^{2}$ Jon Tippin, ${ }^{2}$ Matthew Rizzo ${ }^{3}$ \\ Steven W. Anderson, ${ }^{2}$ \\ ${ }^{1}$ Dept. of Biostatistics, College of Public Health, University of Iowa \\ Iowa City, Iowa, USA \\ ${ }^{2}$ Dept. of Neurology, College of Medicine, University of Iowa \\ Iowa City, Iowa, USA \\ ${ }^{3}$ Dept. of Neurological Sciences, College of Medicine, University of Nebraska Medical Center \\ Omaha, Nebraska, USA \\ E-mail: jeffrey-dawson@uiowa.edu
}

\begin{abstract}
Summary: As part of a study in drivers with obstructive sleep apnea (OSA), we conducted a randomized clinical trial to assess whether individualized feedback can increase compliance with continuous positive airway pressure (CPAP) therapy. After completing 3.5 months of naturalistic driving monitoring, OSA drivers were randomized either to receive an intervention, which was feedback regarding their own naturalistic driving record and CPAP compliance, or to receive no such intervention. In the week immediately after the intervention date, drivers receiving feedback $(n=30)$ improved their CPAP usage by an average of 35.8 minutes per night ( $p=0.008 ; 95 \% \mathrm{CI}=9.6,62.0)$ to a mean level of 296 minutes. By contrast, CPAP usage in the non-feedback group $(n=36)$ decreased an average of 27.5 minutes per night $(\mathrm{p}=0.022 ; 95 \% \mathrm{CI}=4.0,51.0)$ to a mean level of 236 minutes. The mean group-specific changes were higher (better) in the feedback group than in the non-feedback group during the first, second, and third weeks of follow-up ( $p<0.001, p=0.001$, and $p=0.027$, respectively). By weeks 4 through 10 , the effect of the feedback had lost its significance ( $p>0.25$ in all cases). Our study suggests that CPAP compliance can be increased using individualized feedback, but that follow-up feedback sessions or reminders may be necessary for sustained improvement.
\end{abstract}

\section{INTRODUCTION}

Drivers with OSA may have an increased risk of a motor vehicle crash compared to those without the condition (Tregear et al, 2009). Continuous positive airway pressure (CPAP) is a common treatment for OSA that is known to reduce sleepiness in general; however, the effects of CPAP on everyday driving are unclear (Montserrat et al, 2001). Since drivers with OSA may be unaware of the level of their sleepiness (Engleman et al, 1997), we have been studying whether individualized feedback will increase CPAP compliance, which should improve patterns of sleep and alertness during driving. In a previous paper (Krone et al, 2013), we described the method of CPAP data collection and individualized feedback and reported preliminary data on 11 subjects who received the feedback. We now have obtained data on 30 subjects who received the feedback and 36 subjects who did not. The overarching goals of this paper are to 1) test whether the feedback increases CPAP compliance and 2) ascertain the length of time over which improvement is sustained. 


\section{METHOD}

\section{Subjects and study overview}

We have completed our data collection period for a naturalistic driving study targeting 75 subjects with OSA and 55 controls, ages 30-60 years old. At the time of enrollment, all were legally licensed, had 10 or more years of driving experience, used one car for at least $90 \%$ of their driving, and averaged a minimum of 2 hours or 100 miles of driving per week. In the OSA drivers, we monitored approximately 3.5 months of driving, as well as sleep behavior and CPAP compliance. For the clinical trial aspect of this study, we randomized OSA subjects to either a control group or to an intervention group, where the intervention was based on information obtained during the first 3.5 months of observation. We then monitored their CPAP usage over the next 3 months. Data were available for 30 subjects who received feedback and 36 who did not. This study was approved by the University of Iowa Institutional Review Board for Human Subjects Protection.

\section{CPAP initiation and monitoring}

Eligibility for this research study was confirmed using a standard overnight sleep study, followed by an initial clinic visit where subjects received important information about their condition, the results of their sleep study, and instruction on CPAP usage. Approximately one month later, subjects came in for an additional visit, where data were downloaded from the machines for the first time, and information was given regarding their compliance and about sleep hygiene in general. This standard of care was administered to all OSA subjects near the beginning of the study, generally about two months prior to the randomized portion of the study reported herein.

CPAP compliance was measured at home using integrated microprocessors that collected usage data (mask-on times and minutes of usage). Our primary outcome for the measure of CPAP compliance was the total number of minutes used per night. The primary analysis was based on comparing the CPAP compliance between the week before and the week after the intervention date for those who received feedback. For the non-feedback group, we used an analogous "potential intervention date" (i.e., the date that we would have scheduled them to receive the feedback had they been randomized to that group) to define the baseline and post-baseline intervals. As a follow-up analysis, we also looked at data from previous and subsequent weeks, to assess sustainability and stability of the intervention.

\section{Driving monitoring}

Driving behavior was based on monitoring electronic, video, and GPS outputs from an instrumentation package installed in each participant's car (McDonald et al, 2012) over a continuous 3.5-month period ( $\sim 2$ weeks before and 3 months after the initiation of CPAP). The results of this monitoring were not used as an outcome for this clinical trial, but were used to provide individual content for the feedback intervention.

\section{Intervention}


Those receiving the intervention were provided with individualized feedback regarding their CPAP usage, activity level, sleep quality, cognitive test performances, and driving behaviors pertaining to the first 3.5 months of the study. Specifically, each participant met with a neuropsychologist for 45-60 minutes, with spouses encouraged to attend. Regarding driving behavior, videos of three samples of pre-CPAP and three samples of post-CPAP driving were shown to the participant, along with an indication of what types of driving errors had occurred. Each participant also received general information on the dangers of untreated OSA, sleep hygiene guidelines, and the impact of poor sleep on driving safety. The main message was that CPAP usage and good sleep hygiene were likely to lead to better sleep, which is likely to improve cognitive function, which should lead to improved driving performance and safety. For more details and examples regarding the feedback intervention, see Krone et al (2013).

\section{Statistical Analyses}

The average number of minutes of CPAP usage per night per subject was calculated for each one-week period, and this became the unit of analysis. The means and standard deviations of these weekly means were calculated across subjects for each week. The week prior to the feedback intervention date (or the potential intervention date for the non-feedback group) was used as a baseline measure of CPAP compliance. We used a mixed effects linear model to assess the longitudinal trends from baseline ("week 0") through 10 weeks post-baseline. We used contrasts of means from this longitudinal model to make comparisons within groups and between groups. We also graphed the means for the three weeks prior to the intervention.

\section{RESULTS}

Table 1 shows the descriptive and inferential statistics for the CPAP usage. Figure 1 illustrates the longitudinal trends for the two groups, starting three weeks before baseline. The feedback group appears to show a temporary rise in CPAP usage immediately after the intervention, which drops to baseline levels by the fourth week and appears to continue to fall. The asterisks in Table 1 show that week's 1 and 2 have significantly higher means compared to baseline in the feedback group, and a significant decrease at week 7 . The overall trend for the non-feedback group is downward, with the means at weeks $1,2,3,6,7$, and 8 significantly lower than at baseline.

During the baseline period, the mean CPAP usage was similar in the two groups (261 vs. 253 minutes for the feedback group and the non-feedback group, respectively; $p=0.852$ ). This indicates that the randomization was successful. However, when comparing post-baseline means, we subtracted off the group-specific baseline mean so as to be as precise as possible. Note in Figure 1 that the similarities at baseline were also seen for three weeks prior to baseline.

Our primary research hypothesis was supported in that the effect of the feedback intervention was in the positive direction and highly significant $(\mathrm{p}<0.001)$. This effect was stable through the second week post-baseline, but lost about a third of its effect by week 3 (going from about 60 minutes down to 40 minutes). It dropped even further by week 4 and was no longer statistically significant. 
Table 1. Summary of CPAP usage ( $\mathrm{min})$ for the week before and after intervention (* indicates that these means are different than the group-specific baseline mean, $\mathbf{p}<\mathbf{0 . 0 5 0}$ ); post-baseline comparisons between groups are adjusted for baseline values

\begin{tabular}{|c|c|c|c|}
\hline Week & $\begin{array}{c}\text { Mean (SD) CPAP usage } \\
\text { per night in feedback } \\
\text { group (min) } \\
(\boldsymbol{n}=3 \mathbf{3})\end{array}$ & $\begin{array}{c}\text { Mean (SD) CPAP usage per } \\
\text { night in non-feedback group } \\
(\mathbf{m i n}) \\
(\mathbf{n}=36)\end{array}$ & $\begin{array}{c}\text { Mean “feedback minus non- } \\
\text { feedback" difference (95\% } \\
\text { CI), p-value }\end{array}$ \\
\hline $\mathbf{0}$ & $260.7(30.6)$ & $253.0(27.4)$ & $7.7(-74.4,89.8), \mathrm{p}=0.852$ \\
\hline $\mathbf{1}$ & $296.5(29.4)^{*}$ & $225.5(26.4)^{*}$ & $63.3(28.2,98.5), \mathrm{p}<0.001$ \\
\hline $\mathbf{2}$ & $296.4(28.6)^{*}$ & $228.5(25.7)^{*}$ & $60.2(24.7,95.7), \mathrm{p}=0.001$ \\
\hline $\mathbf{3}$ & $268.5(32.4)$ & $220.8(29.0)^{*}$ & $40.0 \quad(4.7,75.3), \mathrm{p}=0.027$ \\
\hline $\mathbf{4}$ & $262.4(31.9)$ & $238.0(28.6)$ & $16.7(-25.0,58.4), \mathrm{p}=0.427$ \\
\hline $\mathbf{5}$ & $240.1(33.5)$ & $245.5(30.1)$ & $-13.1(-51.6,25.3), \mathrm{p}=0.498$ \\
\hline $\mathbf{6}$ & $234.1(33.3)$ & $224.8(29.9)^{*}$ & $1.7(-34.1,37.4), \mathrm{p}=0.927$ \\
\hline $\mathbf{7}$ & $220.0(31.9)^{*}$ & $227.8(28.6)^{*}$ & $-15.4(-53.0,22.1), \mathrm{p}=0.415$ \\
\hline $\mathbf{8}$ & $237.3(33.4)$ & $213.2(30.0)^{*}$ & $16.4(-31.8,64.6), \mathrm{p}=0.500$ \\
\hline $\mathbf{9}$ & $252.6(32.9)$ & $219.2(29.4)$ & $25.7(-29.6,80.9), \mathrm{p}=0.357$ \\
\hline $\mathbf{1 0}$ & $258.6(32.3)$ & $220.8(29.0)$ & $30.2(-26.7,87.1), \mathrm{p}=0.293$ \\
\hline
\end{tabular}

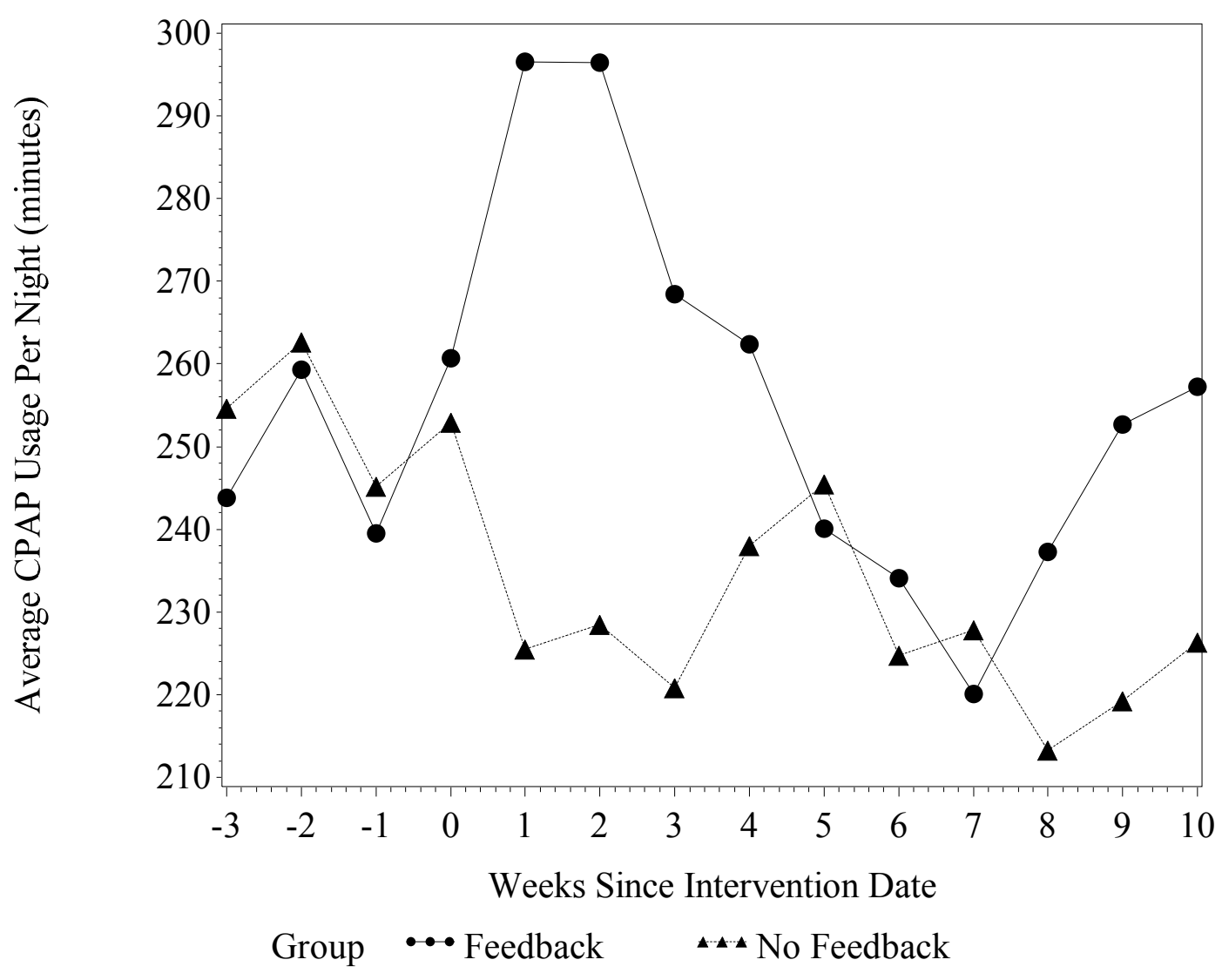

Figure 1. Average minutes of CPAP usage per night, starting 3 weeks prior to intervention baseline (week 0), through 10 weeks post-baseline 


\section{DISCUSSION}

The benefical effects of CPAP on disordered sleep in OSA are well known, as are the effects of disordered sleep on driving. Very little is known regarding the dose-response relationships between CPAP and real world driving safety, and whether demonstrable safety errors in the real world can be used as feedback to improve thaerapeuric compliance with CPAP. This randomized study tested whether the feedback based on real world driving errors and CPAP usage can increase CPAP compliance in OSA drivers, and examined the length of time over which improvement in CPAP compliance is sustained.

The baseline measure of CPAP usage was obtained approximately three months after CPAP began. At that time, the mean usage per night of all subjects combined was 258 minutes, which was just above the recommended target of four hours of CPAP use per night (Weaver et al, 2007). With no intervention, subjects tended to use their CPAP less over time. This may be due to a variety of inconveniences with CPAP, such as discomfort, the noise produced, or potential issues with intimacy. This may explain why even patients who were generally compliant sometimes had nights with zero or near-zero CPAP usage (Krone et al, 2013).

Wolkove et al (2008) reported higher CPAP compliance than we observed, with means of 6.6 hours (396 minutes) per night in long-term compliance. However, it appears that their average did not include the $31 \%$ of their OSA patients who never commenced therapy after disease titration. If their data had included the zero values from these patients, then their compliance average apparently would have been $69 \%$ of 396 minutes, or 273 minutes. This average is still higher than what we usually had in our study, except for weeks 1 and 2 post-intervention in our feedback group.

OSA drivers receiving the individualized feedback responded with a 36-minute-per-night increase in the first week--approximately $14 \%$ higher than the average baseline level. This magnitude of increase was maintained through week 2 , but fell quickly after that. This suggests that some form of recurrent (booster) feedback should be considered. It is not clear whether recurring feedback would be needed indefinitely, or whether the effects of such feedback could be adequately sustained after just a few sessions of repeated feedback. Examining this issue would require additional study.

It is also not clear whether the observed benefit was due to our entire feedback intervention or just a portion thereof. If the main motivator was the general information on the importance of sleep and the links among sleep, alertness, and safe driving, then it may be relatively simple and inexpensive to design a program to alert and remind the subjects of these issues. If the key was the individually-tailored aspect of the feedback, then it would be much more challenging to repeat this feedback multiple times, considering the labor-intensive video review needed to provide examples of driving errors. As a first step in determining the key ingredient in our feedback, it may be advisable to identify subjects who improved the most, and to interview or survey them to obtain this information. 


\section{ACKNOWLEDGMENTS}

We thank the subjects for their willingness to participate in this novel study. This study was supported by NIH R01 HL091917. We also thank all the research staff of the University of Iowa Division of Neuroergonomics for their work.

\section{REFERENCES}

Engleman, H.M., Hirst, W.S.J., \& Douglas, N.J. (1997). Under reporting of sleepiness and driving impairment in patients with sleep apnea/hypopnea syndrome. Journal of Sleep Research, 6, 272-275.

Krone T., Dawson J.D., Anderson S.W., Anderson S.W., Tippin J, \& Rizzo M. (2013). Using feedback from naturalistic driving to improve treatment compliance in drivers with obstructive sleep apnea. Proceedings of Driving Assessment 2013: The Seventh International Driving Symposium on Human Factors in Driving Assessment, Training, and Vehicle Design.

McDonald, A.D., Lee, J.D., Aksan, N.S., Rizzo, M., Dawson, J.D., \& Tippin, J. (2012). Making naturalistic driving data SAX-y. Proceedings of the VTTI Third International Symposium on Naturalistic Driving Research, Blacksburg, Virginia.

Montserrat J.M., Ferrer M., Hernandez L., Farré R., Vilagut G., Navahas D., Badia J.R., Carrasco E., De Pablo J., \& Ballester, E. (2001). Effectiveness of CPAP treatment in daytime function in sleep apnea syndrome: a randomized controlled study with an optimized placebo. Am J Respir Crit Care Med, 164(4), 608-613.

Tregear S., Reston J., Schoelles K., \& Phillips B. (2009). Obstructive sleep apnea and risk of motor vehicle crash: systematic review and meta-analysis. J Clin Sleep Med, 5(6), 573-581.

Weaver T.E., Maislin G., Dinges D.F. Bloxham T., George C.F., Greenberg H., Kader G., Mahowald M., Younger J., \& Al, P. (2007). Relationship between hours of CPAP use and achieving normal levels of sleepiness and daily functioning. SLEEP, 30(6), 711-719.

Wolkove N., Baltzan M., Kamel H., Dabursin R., \& Palayew M. (2008). Long-term compliance with continuous positive airway pressure in patients with obstructive sleep apnea. Can Respir $J, 15(7), 365-369$. 